\title{
Thirring Model with Jump Defect
}

\section{A.R. Aguirre*}

Instituto de Física Teórica, UNESP, São Paulo, Brazil.

E-mail: aleroagueift.unesp.br

\section{J.F. Gomes}

Instituto de Física Teórica, UNESP, São Paulo, Brazil.

E-mail: jfgeift.unesp.br

\section{L.H. Ymai}

Instituto de Física Teórica, UNESP, São Paulo, Brazil.

E-mail: leandroyeift.unesp.br

\section{A.H. Zimerman}

Instituto de Física Teórica, UNESP, São Paulo, Brazil.

E-mail: zimermaneift.unesp.br

\begin{abstract}
The purpose of our work is to extend the formulation of classical affine Toda Models in the presence of jump defects to pure fermionic Thirring model. As a first attempt we construct the Lagrangian of the Grassmanian Thirring model with jump defect (of Backlund type) and present its conserved modified momentum and energy expressions giving a first indication of its integrability.
\end{abstract}

5th International School on Field Theory and Gravitation, April 20 - 242009

Cuiabá city, Brazil

\footnotetext{
* Poster section.
} 


\section{Introduction}

Recently, there has been some interest in the question of whether relativistic integrable field theories admit certain discontinuities (or jump defects) and, if so, what kind of properties they might have [1, 2, 3, 4, 5]. In particular, in [1, 2, 3, 4, 5] using the Lagrangian formulation, it was noted that some bosonic field theories could allow discontinuities like jump defects and yet remain classically integrable. The best understood of these models is the sinh-Gordon in which different soliton solutions are connected in such way that the integrability is preserved.

The defect conditions relating fields evaluated as limits from both sides of the defect turn out to be described by Bäcklund transformation located at the defect. This fact sheds an interesting new light on the role which the Bäcklund transformations have played in the development of soliton theory.

More recently, the extension to classical integrable $N=1$ and $N=2$ super sinh-Gordon models involving bosons and fermions admitting jump defects have been studied [6, 7] using both the Lagrangian approach and the zero curvature formalism. Here we study the Thirring model with jump defects as an example of pure fermionic case. In this work we explicitly construct its modified conserved momentum and energy which gives a very strong suggestion of its classical integrability.

\section{Lagrangian Approach}

The Lagrangian density for Grassmanian Thirring model with jump defect can be written as follows,

$$
\mathscr{L}=\theta(-x) \mathscr{L}_{1}+\theta(x) \mathscr{L}_{2}+\delta(x) \mathscr{L}_{D}
$$

where

$$
\begin{aligned}
\mathscr{L}_{p} & =\frac{i}{2} \psi_{1}^{(p)}\left(\partial_{t}-\partial_{x}\right) \psi_{1}^{\dagger(p)}+\frac{i}{2} \psi_{1}^{\dagger(p)}\left(\partial_{t}-\partial_{x}\right) \psi_{1}^{(p)}+\frac{i}{2} \psi_{2}^{(p)}\left(\partial_{t}+\partial_{x}\right) \psi_{2}^{\dagger(p)}+\frac{i}{2} \psi_{2}^{\dagger(p)}\left(\partial_{t}+\partial_{x}\right) \psi_{2}^{(p)} \\
& +m\left(\psi_{1}^{(p)} \psi_{2}^{\dagger(p)}+\psi_{2}^{(p)} \psi_{1}^{\dagger(p)}\right)-g\left(\psi_{1}^{\dagger(p)} \psi_{2}^{\dagger(p)} \psi_{2}^{(p)} \psi_{1}^{(p)}\right),
\end{aligned}
$$

is the lagrangian density for Thirring model [8] describing massive two-component Dirac fields $\left(\psi_{1}^{(p)}, \psi_{2}^{(p)}\right)$ with $p=1$ corresponding to $x<0, p=2$ corresponding to $x>0$, and $g$ is coupling constant. The defect contribution located at $x=0$ is described by

$$
\begin{aligned}
\mathscr{L}_{D} & =\frac{1}{2}\left[\frac{2 i a}{m} X^{\dagger} \partial_{t} X+i \psi_{1}^{(1)} \psi_{1}^{\dagger(2)}+i \psi_{2}^{(1)} \psi_{2}^{\dagger(2)}-i \psi_{1}^{(2)} \psi_{1}^{\dagger(1)}-i \psi_{2}^{(2)} \psi_{2}^{\dagger(1)}+\right. \\
& +\left(i\left(\psi_{1}^{(2)}-\psi_{1}^{(1)}\right)+a\left(\psi_{2}^{(2)}+\psi_{2}^{(1)}\right)\right) X^{\dagger}+\left(i\left(\psi_{1}^{\dagger(2)}-\psi_{1}^{\dagger(1)}\right)-a\left(\psi_{2}^{\dagger(2)}+\psi_{2}^{\dagger(1)}\right)\right) X \\
& -\frac{i a g}{2 m}\left(i\left(\psi_{1}^{\dagger(2)}-\psi_{1}^{\dagger(1)}\right) \psi_{1}^{(2)} \psi_{1}^{(1)}+a^{-1}\left(\psi_{2}^{\dagger(2)}+\psi_{2}^{\dagger(1)}\right) \psi_{2}^{(2)} \psi_{2}^{(1)}\right) X^{\dagger} \\
& -\frac{i a g}{2 m}\left(-i \psi_{1}^{\dagger(1)} \psi_{1}^{\dagger(2)}\left(\psi_{1}^{(2)}-\psi_{1}^{(1)}\right)+a^{-1} \psi_{2}^{\dagger(1)} \psi_{2}^{\dagger(2)}\left(\psi_{2}^{(2)}+\psi_{2}^{(1)}\right)\right) X \\
& \left.+\frac{a g}{m} \psi_{1}^{\dagger(1)} \psi_{1}^{\dagger(2)} \psi_{1}^{(2)} \psi_{1}^{(1)}+\frac{g}{a m} \psi_{2}^{\dagger(1)} \psi_{2}^{\dagger(2)} \psi_{2}^{(2)} \psi_{2}^{(1)}\right]
\end{aligned}
$$

Here, we introduce auxiliary fields $X$ and $X^{\dagger}$. 
The field equations for $x \neq 0$ are obtained as

$$
\begin{aligned}
i\left(\partial_{t}-\partial_{x}\right) \psi_{1}^{(p)} & =m \psi_{2}^{(p)}+g \psi_{2}^{\dagger(p)} \psi_{2}^{(p)} \psi_{1}^{(p)}, \\
i\left(\partial_{t}+\partial_{x}\right) \psi_{2}^{(p)} & =m \psi_{1}^{(p)}+g \psi_{1}^{\dagger(p)} \psi_{1}^{(p)} \psi_{2}^{(p)}, \\
i\left(\partial_{t}-\partial_{x}\right) \psi_{1}^{\dagger(p)} & =-m \psi_{2}^{\dagger(p)}-g \psi_{1}^{\dagger(p)} \psi_{2}^{\dagger(p)} \psi_{2}^{(p)}, \\
i\left(\partial_{t}+\partial_{x}\right) \psi_{2}^{\dagger(p)} & =-m \psi_{1}^{\dagger(p)}-g \psi_{2}^{\dagger(p)} \psi_{1}^{\dagger(p)} \psi_{1}^{(p)},
\end{aligned}
$$

which are the equations of motion for the Thirring model.

For $x=0$, the equations corresponding to defect conditions are

$$
\begin{aligned}
X & =\left(\psi_{1}^{(2)}+\psi_{1}^{(1)}\right)+\frac{i a g}{2 m}\left(\psi_{1}^{\dagger(2)}+\psi_{1}^{\dagger(1)}\right) \psi_{1}^{(2)} \psi_{1}^{(1)} \\
& =i a^{-1}\left(\psi_{2}^{(2)}-\psi_{2}^{(1)}\right)-\frac{g}{2 a^{2} m}\left(\psi_{2}^{\dagger(2)}-\psi_{2}^{\dagger(1)}\right) \psi_{2}^{(2)} \psi_{2}^{(1)}
\end{aligned}
$$

and its hermitian conjugated

$$
\begin{aligned}
X^{\dagger} & =\left(\psi_{1}^{\dagger(2)}+\psi_{1}^{\dagger(1)}\right)-\frac{i a g}{2 m}\left(\psi_{1}^{(2)}+\psi_{1}^{(1)}\right) \psi_{1}^{\dagger(1)} \psi_{1}^{\dagger(2)} \\
& =-i a^{-1}\left(\psi_{2}^{\dagger(2)}-\psi_{2}^{\dagger(1)}\right)-\frac{g}{2 a^{2} m}\left(\psi_{2}^{(2)}-\psi_{2}^{(1)}\right) \psi_{2}^{\dagger(1)} \psi_{2}^{\dagger(2)}
\end{aligned}
$$

together with

$$
\begin{aligned}
\partial_{t} X & =\frac{m a^{-1}}{2}\left(\psi_{1}^{(2)}-\psi_{1}^{(1)}\right)-\frac{i m}{2}\left(\psi_{2}^{(2)}+\psi_{2}^{(1)}\right) \\
& -\frac{g}{4}\left(i\left(\psi_{1}^{\dagger(2)}-\psi_{1}^{\dagger(1)}\right) \psi_{1}^{(2)} \psi_{1}^{(1)}+a^{-1}\left(\psi_{2}^{\dagger(2)}+\psi_{2}^{\dagger(1)}\right) \psi_{2}^{(2)} \psi_{2}^{(1)}\right), \\
\partial_{t} X^{\dagger} & =\frac{m a^{-1}}{2}\left(\psi_{1}^{\dagger(2)}-\psi_{1}^{\dagger(1)}\right)+\frac{i m}{2}\left(\psi_{2}^{\dagger(2)}+\psi_{2}^{\dagger(1)}\right) \\
& +\frac{g}{4}\left(i \psi_{1}^{\dagger(1)} \psi_{1}^{\dagger(2)}\left(\psi_{1}^{(2)}-\psi_{1}^{(1)}\right)-a^{-1} \psi_{2}^{\dagger(1)} \psi_{2}^{\dagger(2)}\left(\psi_{2}^{(2)}+\psi_{2}^{(1)}\right)\right)
\end{aligned}
$$

Notice that we can also write

$$
\begin{aligned}
X & =\left(\psi_{1}^{(2)}+\psi_{1}^{(1)}\right)+\frac{i a g}{2 m} \psi_{1}^{(2)} \psi_{1}^{(1)} X^{\dagger} \\
& =\left(\psi_{1}^{(2)}+\psi_{1}^{(1)}\right)+\frac{i a g}{2 m} \psi_{1}^{(1)} X^{\dagger} X \\
& =\left(\psi_{1}^{(2)}+\psi_{1}^{(1)}\right)-\frac{i a g}{2 m} \psi_{1}^{(2)} X^{\dagger} X
\end{aligned}
$$

and similarly for $X^{\dagger}$. As a consequence of (2.10), (2.11) and the Thirring equations of motion (2.4) - (2.7), we obtain

$$
\begin{aligned}
& \left(\partial_{t}+\partial_{x}\right) X=m a^{-1}\left(\psi_{1}^{(2)}-\psi_{1}^{(1)}\right)-\frac{i g}{2}\left(\psi_{1}^{\dagger(2)}-\psi_{1}^{\dagger(1)}\right) \psi_{1}^{(2)} \psi_{1}^{(1)} \\
& \left(\partial_{t}-\partial_{x}\right) X=-i m\left(\psi_{2}^{(2)}+\psi_{2}^{(1)}\right)-\frac{g}{2 a}\left(\psi_{2}^{\dagger(2)}+\psi_{2}^{\dagger(1)}\right) \psi_{2}^{(2)} \psi_{2}^{(1)}
\end{aligned}
$$

which can be re-written as

$$
\begin{aligned}
& \left(\partial_{t}+\partial_{x}\right) X=m a^{-1} X-2 m a^{-1} \psi_{1}^{(1)}-i g \psi_{1}^{\dagger(1)} \psi_{1}^{(1)} X-i g X^{\dagger} X \psi_{1}^{(1)} \\
& \left(\partial_{t}-\partial_{x}\right) X=-m a X-2 m i \psi_{2}^{(1)}-i g \psi_{2}^{\dagger(1)} \psi_{2}^{(1)} X-g a X^{\dagger} X \psi_{2}^{(1)} .
\end{aligned}
$$


The equations $(2.8,2.9,2.12,2.13,2.14)$ are the Bäcklund transformation for the classical anticommuting Thirring model [9]. In other words, in this approach the Bäcklund transformation at $x=0$ represents the boundary conditions between two regions.

\section{Conserved Momentum and Energy}

The canonical momentum (which is not expected to be preserved) is given by

$$
\begin{aligned}
P= & \int_{-\infty}^{0} d x\left[\frac{i}{2}\left(\psi_{1}^{(1)} \partial_{x} \psi_{1}^{\dagger(1)}+\psi_{1}^{\dagger(1)} \partial_{x} \psi_{1}^{(1)}+\psi_{2}^{(1)} \partial_{x} \psi_{2}^{\dagger(1)}+\psi_{2}^{\dagger(1)} \partial_{x} \psi_{2}^{(1)}\right)\right] \\
& +\int_{0}^{\infty} d x\left[\frac{i}{2}\left(\psi_{1}^{(2)} \partial_{x} \psi_{1}^{\dagger(2)}+\psi_{1}^{\dagger(2)} \partial_{x} \psi_{1}^{(2)}+\psi_{2}^{(2)} \partial_{x} \psi_{2}^{\dagger(2)}+\psi_{2}^{\dagger(2)} \partial_{x} \psi_{2}^{(2)}\right)\right] .
\end{aligned}
$$

Using the field equations $(2.4,2.5,2.6,2.7)$ we obtain

$$
\begin{aligned}
\frac{d P}{d t}= & {\left[m\left(\psi_{1}^{(1)} \psi_{2}^{\dagger(1)}+\psi_{2}^{(1)} \psi_{1}^{\dagger(1)}\right)-g \psi_{1}^{\dagger(1)} \psi_{2}^{\dagger(1)} \psi_{2}^{(1)} \psi_{1}^{(1)}+\frac{i}{2}\left(\psi_{1}^{\dagger(1)} \partial_{t} \psi_{1}^{(1)}+\psi_{1}^{(1)} \partial_{t} \psi_{1}^{\dagger(1)}\right.\right.} \\
& \left.\left.+\psi_{2}^{\dagger(1)} \partial_{t} \psi_{2}^{(1)}+\psi_{2}^{(1)} \partial_{t} \psi_{2}^{\dagger(1)}\right)\right]_{x=0}-\left[m\left(\psi_{1}^{(2)} \psi_{2}^{\dagger(2)}+\psi_{2}^{(2)} \psi_{1}^{\dagger(2)}\right)-g \psi_{1}^{\dagger(2)} \psi_{2}^{\dagger(2)} \psi_{2}^{(2)} \psi_{1}^{(2)}\right. \\
& \left.+\frac{i}{2}\left(\psi_{1}^{\dagger(2)} \partial_{t} \psi_{1}^{(2)}+\psi_{1}^{(2)} \partial_{t} \psi_{1}^{\dagger(2)}+\psi_{2}^{\dagger(2)} \partial_{t} \psi_{2}^{(2)}+\psi_{2}^{(2)} \partial_{t} \psi_{2}^{\dagger(2)}\right)\right]_{x=0}
\end{aligned}
$$

Considering the boundary conditions $(2.8,2.9,2.13,2.14)$ the right hand side becomes a total time derivative. Thus, we found a functional $P_{D}$ given by

$$
\begin{aligned}
P_{D}= & \frac{i a}{m}\left(X \partial_{t} X^{\dagger}-\left(\partial_{t} X\right) X^{\dagger}\right)-\frac{i}{2}\left(\psi_{1}^{(1)} \psi_{1}^{\dagger(2)}+\psi_{1}^{\dagger(1)} \psi_{1}^{(2)}+3 \psi_{2}^{(1)} \psi_{2}^{\dagger(2)}+3 \psi_{2}^{\dagger(1)} \psi_{2}^{(2)}\right) \\
& -\frac{g a}{2 m} \psi_{1}^{\dagger(1)} \psi_{1}^{\dagger(2)} \psi_{1}^{(2)} \psi_{1}^{(1)}+\frac{g}{2 m a} \psi_{2}^{\dagger(1)} \psi_{2}^{\dagger(2)} \psi_{2}^{(2)} \psi_{2}^{(1)},
\end{aligned}
$$

so that $\mathscr{P}=P+P_{D}$ is conserved. This 'modified' momentum $\mathscr{P}$ appears to be a 'total' momentum which is preserved containing bulk and defect contributions. In the case of the energy

$$
\begin{aligned}
E= & \int_{-\infty}^{0} d x\left[\frac{i}{2}\left(\psi_{1}^{(1)} \partial_{x} \psi_{1}^{\dagger(1)}+\psi_{1}^{\dagger(1)} \partial_{x} \psi_{1}^{(1)}-\psi_{2}^{(1)} \partial_{x} \psi_{2}^{\dagger(1)}-\psi_{2}^{\dagger(1)} \partial_{x} \psi_{2}^{(1)}\right)\right. \\
& \left.-m\left(\psi_{1}^{(1)} \psi_{2}^{\dagger(1)}+\psi_{2}^{(1)} \psi_{1}^{\dagger(1)}\right)+g \psi_{1}^{\dagger(1)} \psi_{2}^{\dagger(1)} \psi_{2}^{(1)} \psi_{1}^{(1)}\right]+\int_{0}^{\infty} d x[(1) \leftrightarrow(2)],
\end{aligned}
$$

the energy-like conserved quantity is $\mathscr{E}=E+E_{D}$, where

$$
\begin{aligned}
E_{D}= & \frac{i a}{m}\left(X \partial_{t} X^{\dagger}-\left(\partial_{t} X\right) X^{\dagger}\right)-\frac{i}{2}\left(\psi_{1}^{(1)} \psi_{1}^{\dagger(2)}+\psi_{1}^{\dagger(1)} \psi_{1}^{(2)}+\psi_{2}^{(1)} \psi_{2}^{\dagger(2)}+\psi_{2}^{\dagger(1)} \psi_{2}^{(2)}\right) \\
& -\frac{g a}{2 m} \psi_{1}^{\dagger(1)} \psi_{1}^{\dagger(2)} \psi_{1}^{(2)} \psi_{1}^{(1)}-\frac{g}{2 m a} \psi_{2}^{\dagger(1)} \psi_{2}^{\dagger(2)} \psi_{2}^{(2)} \psi_{2}^{(1)} .
\end{aligned}
$$


We notice that this functional $E_{D}$ appears on-shell to be the defect lagrangian $\mathscr{L}_{D}$. This property already was found in the sinh-Gordon model with defect [2]. Then, in spite of the loss of translation invariance, the fields can exchange both energy and momentum with the defect.

In this work we have studied the classical integrability of the Grassmanian Thirring model with jump defect by constructing the lowest conserved quantities, namely, the modified momentum and energy. The integrability of the model involves also higher conservation laws which are encoded within the Lax pair formalism. This work is in progress.

\section{Acknowledgments}

We would like to thank the organisers of the FIFTH INTERNATIONAL SCHOOL ON FIELD THEORY AND GRAVITATION for the opportunity to present these ideas. ARA and LHY thank FAPESP, AHZ and JFG CNPq for financial support.

\section{References}

[1] S. Ghosal and A. Zamolodchikov, Int. J. Mod. Physics A 9 (1994). 3841, [hep-th/9306002].

[2] P. Bowcock, E. Corrigan and C. Zambon, Int. J. Mod. Physics A 19 (Supplement) (2004) 82-91, [hep-th/0305022].

[3] P. Bowcock, E. Corrigan and C. Zambon, J. of High Energy Physics JHEP (2004) 056, [hep-th/0401020].

[4] E. Corrigan and C. Zambon, J. of Physics A37 (2004) L471.

[5] P. Bowcock, E. Corrigan and C. Zambon, J. of High Energy Physics JHEP (2004) 023, [hep-th/0506169].

[6] J.F. Gomes, L.H Ymai and A.H. Zimerman, J. Physics A39 (2006) 7471, [hep-th/0601014].

[7] J.F. Gomes, L.H Ymai and A.H. Zimerman, JHEP03 (2008) 001.

[8] W. Thirring, Ann. Phys. (NJ) 3, 91 (1958).

[9] A. Izergin and J. Stehr, DESY 76/60, Nov 1976. 8pp. 